\title{
\& Research Square \\ Metabolomic Profiling of Five Hulless Barley with Different Seed Coat Colors
}

\author{
Lijing Liang ( $\sim$ 43228795@qq.com ) \\ Sichuan Agricultural University https://orcid.org/0000-0002-0875-7601 \\ Wenbo Li \\ Sichuan Agricultural University \\ Min Tian \\ Sichuan Agricultural University \\ Jiajia Pan \\ Sichuan Agricultural University \\ Zongyun Feng \\ Sichuan Agricultural University
}

\section{Research Article}

Keywords: Colored hulless barley, metabolites, PCA, OPLS-DA, UPLC-ESI-MS/MS

Posted Date: October 27th, 2021

DOI: https://doi.org/10.21203/rs.3.rs-839743/v1

License: (c) (1) This work is licensed under a Creative Commons Attribution 4.0 International License. Read Full License 


\section{Abstract}

Colored hulless barley (Hordeum vulgare L.) is a high-quality germplasm resource that is rich in nutrients, such as protein, $\beta$-glucan, flavonoids, amino acids, vitamins, microelements, and dietary fiber. However, a systematic evaluation of the metabolites present in colored cultivars is lacking. This study employed an untargeted metabolomics approach using ultra-high-performance liquid chromatography-tandem mass spectrometry (LC-MS) to analyze the metabolic profiles of five hulless barley cultivars with different grain colors. Six-hundred and eight metabolites in various chemical categories were detected; ABC transporters, flavonoid biosynthesis, and anthocyanin biosynthesis were found to be the most significant metabolic pathways. Principal component analysis and orthogonal partial least squares-discriminant analysis revealed significant metabolic differences among the samples, and the colored barley cultivars could be separated from white barley. Black, blue, and purple grains rich in natural antioxidants were identified as promising ingredients for the development of cereal-based functional foods. These findings showed that the nutritional function and quality of hulless barley were strongly correlated with its natural color, thereby improving our understanding of the metabolic mechanisms and functional health value associated with different seed coat colors in different hulless barley cultivars. Our results thus provide a theoretical basis for the future study of hulless barley-related products.

\section{Introduction}

Barley (Hordeum vulgare L.) is a globally cultivated grain that is currently ranked fifth in the world in terms of dry matter production (Roljević et al. 2020). Hulless barley (H. vulgare var. nudum Hook. f.) is a cultivar of barley with a lemma in the bare grain that is separated from the caryopsis, which is why it is referred to as hulless. Hulless barley is the only crop that can be cultivated in the Tibetan plateau and is thus the main staple food crop for Tibetans as well as an important feed for livestock (Ramakrishna et al. 2017; Zeng et al. 2015). Hulless barley is valued globally for its significant nutritional value, including protein, crop for Tibetans, amino acids, various vitamins, trace elements (zinc and selenium), and dietary fiber (Idehen et al. 2017; Yang et al. 2018).

Seed coat color is an important agronomic crop trait because of its unique biological activity and healthcare functions (Yao et al. 2018). The breeding of colored barley varieties has become increasingly prevalent in agriculture in recent years. Purple, blue, and black grains are considered as promising ingredients for developing functional grain-based foods, as they are a natural source of antioxidants, are rich in phenolic compounds called anthocyanins, and contain essential amino acids (Abdel et al. 2003; Abdel et al. 2006; Shen et al. 2018; Zeven, 1991). At maturity, the barley grain may have different pigmentation, which is formed by the pigment deposited in the seed coat and aleurone layer. Studies have shown that the natural pigments in colored barley, which include anthocyanins, essential amino acids, and trace elements, are generally higher than those of ordinary white barley and have significant physiological functions. 
Therefore, there has been increasing focus on the utilization of colored barley cultivars (Kim et al. 2007; Lin et al. 2018). The most studied seed coat colors are yellow, purple, red, and blue, which are thought to be associated with different subgroups of flavonoids (Shoeva et al. 2016).

The seed coat of barley is yellow due to the synthesis of proanthocyanidins in the seed coat (testa layer) (Aastrup et al. 1984); the anthocyanins synthesized in the pericarp and glume are related to red and purple seed coats (Zhang et al. 2019); and anthocyanins synthesized in the aleurone layer of the grain result in a blue seed coat (Harlan. 1914). These pigments are not present in the white barley grains (Shoeva et al. 2016). Colored hulless barley is a high-quality germplasm resource, and its nutritional function and nutritional quality are strongly correlated with its natural color (Ge et al. 2021; Yan et al. 2009; Yao et al. 2018). Qualitative and quantitative variations in the phytochemical profiles and nutrients, and especially the bioactive compounds of hulless barley, are believed to contribute to the differences in its health-promoting properties (Zhang et al. 2019). Colored barley varieties have been found to be associated with greater beneficial health properties than the standard (white) cultivar because they produce pearling fractions that are rich in bioactive compounds (Bellido et al. 2009; Chen et al. 2013; Dawson et al. 2015; Fernie et al, 2009; Khakimov et al. 2014).

Metabolomics is a powerful tool that is widely used for the large-scale detection of small-molecule metabolites and is convenient for detecting hundreds of metabolites in cells at a specific time point, thus offering insight into the phenotype (Chen et al. 2013; Dawson et al. 2015; Fernie et al. 2009; Khakimov et al. 2014). There has been research interest in the metabolites present in hulless barley due to their unique nutritional characteristics and promising health benefits(Jung et al. 2000; Khlestkina 2013). However, specific compounds have been studied mainly through targeted metabolomics approaches, rather than by thoroughly investigating all the phytochemical constituents present in hulless barley. For instance, the flavonoid biosynthesis metabolic pathway has been well-studied (Badbro. 1993; Lin et al. 2018; Suriano et al. 2020). To the best of our knowledge, a systematic evaluation and comparison of the metabolites present in different colored hulless barley cultivars is lacking. The accumulation of metabolites differs in different tissues, species, as well as temporally (Dong et al. 2014; Wang et al. 2016). In this study, five different colored hulless barley varieties were collected from the main barley cultivation areas of China. An untargeted metabolomics approach using ultra-high-performance liquid chromatography-tandem mass spectrometry (UHPLC-MS/MS) was used to analyze the metabolic profiles and variations in phytochemicals among these five different colored hulless barley varieties. Our aim was to contribute toward an elucidation of the metabolic differences among different colored hulless barley cultivars and provide a reference for further nutritional applications of colored hulless barley.

\section{Materials And Methods}

\section{Plant materials}

The white cultivar '1127' and blue cultivar 'xiaolanqingke' (XLQK) were collected from Tibet; the blue cultivar 'ganzilan' (GZL) and black-purple cultivar 'ganzi_160505' (GZ-160505) were collected from Ganzi 
Prefecture in Sichuan Province of China; and the purple cultivar 'beiqing_2' (BQ_2) was collected from Haibei Prefecture in Qinghai Province (Fig. 1A). When the plants were fully mature, they were harvested and dried. Once fully dried, the plants were threshed indoors, and the mature seeds were harvested and stored at $-80^{\circ} \mathrm{C}$ until further analysis. Grain color was determined by scanning with a color difference meter (Konica Minolta CM-5).

\section{Sample preparation and extraction for metabolomic analysis}

The freeze-dried hulless barley seeds were crushed to a powder with a mixer mill (MM 400, Retsch) (30 $\mathrm{Hz}, 1.5 \mathrm{~min}$ ). One hundred milligrams of powder were weighed and dissolved in $0.6 \mathrm{~mL} 70 \%$ methanol extract and then dissolved at $4^{\circ} \mathrm{C}$ overnight. The samples were stored in a bottle for UPLC-MS/MS analysis following centrifugation at $10,000 \mathrm{~g}$ for $10 \mathrm{~min}$ and then filtered through a $0.22 \mu \mathrm{m}$ pore membrane.

\section{High-performance liquid chromatography (HPLC) conditions}

The data acquisition instrument system included an UPLC-ESI-MS/MS system (UPLC, Shim-pack UFLC SHIMADZU CBM30A system, www.shimadzu.com.cn/; MS, Applied Biosystems, 4500 Q TRAP, www.appliedbiosystems.com.cn/). A Waters ACQUITY UPLC HSS T3 C18 column $(1.8 \mu \mathrm{m}, 2.1 \mathrm{~mm} * 100$ $\mathrm{mm}$ ) was used. The mobile phase consisted of solvent $A$ (pure water with $0.04 \%$ acetic acid) and solvent $\mathrm{B}$ (acetonitrile with $0.04 \%$ acetic acid). The gradient program was $95: 5 \mathrm{~V} / \mathrm{V}$ at $0 \mathrm{~min}$, with a linear gradient to $5: 95 \mathrm{~V} / \mathrm{V}$ over $10 \mathrm{~min}$, where it was held for $1 \mathrm{~min}$ at 5:95 V/V at $11.0 \mathrm{~min}$, following which it was adjusted to $95 \%$ A $5.0 \%$ within $0.10 \mathrm{~min}$, where it was held for $2.9 \mathrm{~min}$. The temperature was $40^{\circ} \mathrm{C}$ and the injection volume was $4 \mu \mathrm{L}$. The effluent was alternatively connected to an ESI-triple quadrupole-linear ion trap (QTRAP)-MS.

\section{ESI-QTRAP-MS/MS}

Linear ion trap (LIT) and triple quadrupole (QQQ) scans were acquired on a 4500 QTRAP equipped with an electrospray ionization (ESI) Turbo lon-Spray interface operating in positive and negative ionization mode and controlled by Analyst 1.6 .3 software (AB Sciex).

The ESI conditions mainly included: electrospray ionization temperature, $550^{\circ} \mathrm{C}$; spray voltage (IS), 5500 V); and ion source curtain gas (CUR), $30 \mathrm{psi}$. The collys-activated dissociation (CAD) parameter was set to high. In QQQ, each ion pair was scanned based on the optimized declustering potential (DP) and collision energy (CE).

\section{Qualitative and quantitative analysis of metabolites}

Qualitative analyses of metabolites were carried out using the self-built database MWDB (Metware Biotechnology Co., Ltd. Wuhan, China) and public database of metabolite information. The isotopic 
signals, the repeated signals of $\mathrm{K}+$ ions, $\mathrm{Na}+$ ions, $\mathrm{NH} 4+$ ions, and the repeated signals of fragments of other larger molecular weight substances were removed. Metabolites were quantified using the MRM model. The mass spectrum peak area of all substances was integrated, and the mass spectrum peak of the same metabolite in different samples was integrated and corrected (Markou \& Kassomenos. 2010).

\section{Statistical analysis}

The seed coat color differences were evaluated by cluster analysis. Cluster analysis is an analytical method that divides samples into several categories by measuring the similarity degree of the samples, ultimately aggregating them into one class step-by-step (Carlos et al. 2010). The color difference value of the hulless barley cultivars was used as the input into the cluster analysis.

All metabolites were analyzed using R software (www.r-project.org). Principal Component Analysis (PCA), hierarchical clustering analysis (HCA), and orthogonal partial least squares-discriminant analysis (OPLSDA) were used to analyze the metabolic data according to previously described methods (Wang et al. 2016). The data were unit variance-scaled prior to the unsupervised PCA.

The Pearson's correlation coefficient (PCC) between the samples was calculated using the 'cor' function in $\mathrm{R}$, and the HCA results of the metabolites were presented in the form of a heatmap with dendrogram.

Variable importance in the projection (VIP) values extracted from the OPLS-DA results $\geq 1$ and absolute Log2FC (fold change) $\geq 1$ were used to determine the differentially abundant metabolites between groups. The data were log-transformed (log2) and mean-centered prior to OPLS-DA. A permutation test (200 permutations) was performed to confirm that the OPLS-DA model had not been overfitted.

The biological significance associated with hulless barley color was further evaluated through the functional analysis of metabolic pathways using the Kyoto Encyclopedia of Genes and Genomes (KEGG) compound database (http://www.kegg.jp/kegg/compound/) to annotate identified metabolites, and the annotated metabolites were then mapped to the KEGG pathway database (http://www.kegg.jp/kegg/pathway.html). Pathways with significantly regulated metabolites were then fed into MSEA (metabolite sets enrichment analysis), and their significance was determined based on the $P$-values of the hypergeometric test.

\section{Results And Discussion}

\section{Analysis of color differences in hulless barley}

The color differences in the seed coat of hulless barley are shown as * $L * a * b$ ( $L *$ represents the brightness, a* represents the red-green axis, and b* represents the blue-yellow axis) in Table 1, which reflects the significant differences in the color characteristics of the five hulless barley cultivars (Leónet al, 2006). Analysis of the color value showed that the brightness factor $L^{*}$, red factor $a^{*}$, and blue factor $b^{\star}$ decreased from white hulless barley to colored hulless barley. In the cluster analysis (Fig. 1B), the white hulless barley could be clearly distinguished from the colored hulless barley varieties. 
Table 1

Color values of the seed coats of five hulless barley cultivars

\begin{tabular}{|llllll|}
\hline Number & Cultivar & Seed coat color & L & a & b \\
\hline 1 & 1127 & white & $56.27 \pm 0.13$ & $8.52 \pm 0.26$ & $24.83 \pm 0.88$ \\
\hline 2 & BQ-2 & purple & $47.49 \pm 0.62$ & $3.61 \pm 0.13$ & $15.32 \pm 0.12$ \\
\hline 3 & GZL & blue & $49.14 \pm 0.72$ & $2.27 \pm 0.15$ & $12.94 \pm 0.27$ \\
\hline 4 & XLQK & blue & $46.17 \pm 0.33$ & $5.81 \pm 0.28$ & $19.34 \pm 0.12$ \\
\hline 5 & GZ-160505 & black-purple & $36.61 \pm 0.45$ & $5.03 \pm 0.25$ & $8.37 \pm 0.45$ \\
\hline
\end{tabular}

\section{Metabolic profiling}

In total, 608 metabolites were detected (Supplementary Table S1), including 147 flavonoids, 87 amino acids and derivatives, 78 phenolic acids, 74 lipids, 47 organic acids, 46 nucleotides and derivatives, 42 alkaloids, 10 lignans and coumarins, eight tannins, four terpenoids, and 71 others. The clustering heatmap of the metabolites (Fig. 2) showed there were significant differences in the metabolite levels of the five cultivars, with the colored hulless barley varieties being clearly distinguished from the white cultivar. This finding was generally consistent with the dendrogram based on the color difference values (Fig. 1b). GZL and BQ_2, although cultivated in different regions, grouped into one category according to seed coat color and possessed similar metabolite contents. The white cultivar 1127 could be clearly distinguished from the other cultivars. This finding was demonstrated by clustering analysis of the samples and showed that the metabolites of hulless barley were strongly related to seed coat color.

\section{Differential metabolite analysis using PCA}

The first two principal components of the PCA score plot were responsible for $51.31 \%$ (28.68\% for PC1 and $22.63 \%$ for PC2) of the variation in the metabolite profiles. There was a clear separation of these five cultivars, suggesting that each group had a relatively distinct metabolic profile. The distinct separation of 1127 (white hulless barley) on the left side of the plot from the other four varieties, which clustered on the right side of the plot, confirmed the distinction of white hulless barley from the colored cultivars. The HCA and PCA results suggested that the differences in the metabolite contents might be responsible for the differences in the seed coat color of hulless barley.

\section{Differential metabolite analysis using OPLS-DA}

OPLS-DA is a multivariate statistical analysis method for supervised pattern recognition that can maximize the differentiation between groups and identify differential metabolites. In this study, OPLS-DA was carried out to further verify the significantly different metabolites between the five hulless barley cultivars. 
High predictability (Q2) and strong goodness-of-fit (R2X, R2Y) of the OPLS-DA models were observed for comparisons between 1127 and $X L Q K(R 2 X=0.771, R 2 Y=1, Q 2=0.989$; Fig. 4A), 1127 and GZ_160505 $(R 2 X=0.776, R 2 Y=1, Q 2=0.989 ;$ Fig. 4B), 1127 and GZL (R2X = 0.773, R2Y = 1, Q2 = 0.987; Fig. 4C), 1127 and BQ_2 (R2X = 0.768, R2Y = 1, Q2 = 0.986; Fig. 4D), XLQK and BQ_2 (R2X = 0.744, R2Y = 1, Q2 = 0.979; Fig. 4E), XLQK and GZL (R2X = 0.763, R2Y = 1, Q2 = 0.987; Fig. 4F), XLQK and GZ_160505 (R2X = 0.748, $R 2 Y=1, Q 2=0.982$; Fig. 4G), GZL and BQ_2 (R2X = 0.715, R2Y = 1, Q2 = 0.976; Fig. 4H), GZL and GZ_160505 (R2X = 0.748, R2Y = 1, Q2 = 0.982; Figure; 4I), and BQ_2 and GZ_160505 (R2X = 0.748, R2Y = 1, $\mathrm{Q} 2=0.982 ;$ Figure; $4 \mathrm{~J}$ ). The validation of the OPLS-DA models presented a high interpretation rate (R2Y) and prediction degree $(\mathrm{Q} 2>0.9)$, indicating that the models were appropriate.

\section{Differential metabolite screening, functional annotation, and enrichment analysis}

The differential metabolites among the five cultivars were screened using a fold-change ( $\geq 2$ or $\leq 0)$ and variable importance in the projection (VIP) value $(\geq 1)$ of the OPLS-DA model. The screening results are shown in Supplementary Table S2 and are illustrated using volcano plots (Fig. 5A-D) and Venn diagrams (Fig. 5E, F). There were 181 significantly different metabolites between 1127 and BQ_2 (134 downregulated, 47 up-regulated), 219 between 1127 and GZ_160505 (115 down-regulated, 104 up-regulated), 207 between 1127 and GZL (131 down-regulated, 76 up-regulated), and 246 between 1127 and XLQK (146 down-regulated, 100 up-regulated). Compared with white hulless barley, there were more downregulated metabolites in the colored hulless barley, and these metabolites could clearly distinguish the colored hulless barley from the white hulless barley. The screening results also showed that, in total, about one third of the differentially abundant metabolites were flavonoids (62/181 in 1127 vs. BQ_2; $84 / 219$ in 1127 vs. GZ_160505; 65/207 in 1127 vs. GZL; and 76/246 in 1127 vs. XLQK). These metabolites were mainly flavonoids and may constitute the representative differential metabolites for the different colored varieties. A difference in phenolic compound composition with grain color was previously observed in both barley and hulless barley (Abdel et al. 2012), which corroborates our results.

Using the intersection of each comparison group in the Venn diagram (Fig. 5E), a total of 70 common differential metabolites were observed, and each comparison group possessed unique differential metabolites. The Venn diagram in Fig. $5 \mathrm{~F}$ shows the overlapping and unique metabolites among the comparison group. There were 28 metabolites that were only present in the colored hulless barley, 19 of which were flavonoids and three of which were phenolic acids. These metabolites were primarily flavonoids and may be considered as representative differential metabolites for colored hulless barley.

To gain further insight into the differences in flavonoids between colored and white hulless barley, differential flavonoids among the comparison groups were compared (Supplementary Table 3). The results showed that, except for BQ_2 (26/62 in 1127 vs. BQ_2), most of the flavonoids (69/84 in 1127 vs. GZ_160505; 47/65 in 1127 vs. GZL; and 62/76 in 1127 vs. XLQK) were up-regulated as the color of the seed coat changed from white to purple-black. Many flavonoids were up-regulated in the colored hulless barley, indicating that colored hulless barley is rich in flavonoids. 
Based on the intersection of each comparison group (Supplementary Table 3), 14 common differential flavonoid metabolites were up-regulated among the comparison groups 1127 vs. BQ_2, 1127 vs. GZ_160505, 1127 vs. GZL, and 1127 vs. XLQK. Rutin was detected in all the samples, though the differences among the colored hulless barley and white cultivar were nonsignificant. Except for cyanidin 3,5-0-diglucoside and peonidin 3-0-glucoside in BQ_2, most of the anthocyanins were up-regulated in the colored varieties compared with the control. Isoflavones are generally exclusively present in legumes, such as soybeans, and play important roles in plant defense and nodulation (Jung et al. 2000; Kim et al. 2007; Shoeva et al. 2016). In the present study, three isoflavones were detected in all hulless barley varieties, of which afzelechin(3,5,7,4'-tetrahydroxyflavan) was down-regulated and 2'-hydroxyisoflavone was up-regulated, whereas aracarpene 2 was down-regulated in BQ_2 and up-regulated in GZ_160505 and XLQK.

\section{Differential metabolic pathways among the samples}

The differentially abundant metabolites among the five hulless barley cultivars were annotated using the KEGG database (http://www.genome.jp/kegg/) in order to obtain the detailed pathway information (Fig. 6A-D, Supplementary Table S4). The KEGG classification results and enrichment analyses indicated that the majority of differential metabolites were involved in metabolic pathways, including metabolic pathways (44/84 [52.38\%], 1127 vs. BQ_2; 45/86 [52.33\%] in 1127 vs. GZ_160505; 57/95 [60\%] in 1127 vs. GZL; 65/113 [57.52\%] in 1127 vs. XLQK), biosynthesis of secondary metabolites $(22 / 84$ [26.19\%] in 1127 vs. BQ_2; 23/86 [26.74\%] in 1127 vs. GZ_160505; $27 / 95$ [28.42\%] in 1127 vs. GZL; $34 / 113$ [30.09\%] in 1127 vs. XLQK); ABC transporters (12/84 [14.29\%] in 1127 vs. BQ_2; 8/86 [9.30\%] in 1127 vs. GZ_160505; 14/95 [14.74\%] in 1127 vs. GZL; 19/113 [16.81\%] in 1127 vs. XLQK); flavonoid biosynthesis (9/84 [10.71\%] in 1127 vs. BQ_2; 13/86 [15.12\%] in 1127 vs. GZ_160505; 7/95 [7.37\%] in 1127 vs. GZL; $13 / 113$ [11.50\%] in 1127 vs. XLQK); and anthocyanin biosynthesis (5/84 [5.95\%] in 1127 vs. BQ_2; 6/86 [6.98\%] in 1127 vs. GZ_160505; 6/95 [6.32\%] in 1127 vs. GZL; 6/113 [5.31\%] in 1127 vs. XLQK).

Enrichment analysis (Fig. 6A-D) also indicated that the differential metabolites of the comparison groups were mainly involved in flavonoid biosynthesis, flavone and flavonol biosynthesis, and anthocyanin biosynthesis.

\section{Conclusions}

In the present study, we focused on the metabolic diversity, particularly flavonoids, of five hulless barley varieties and annotated 608 metabolites, including flavones, amino acids, and phenolic acids. The metabolic profiles of the colored hulless barley varieties could be effectively discriminated from that of the white cultivar, and the differences were mainly attributed to metabolites associated with $A B C$ transporters, flavonoid biosynthesis, and anthocyanin biosynthesis pathways. In addition, 18 flavonoids and three phenolic acids were detected and quantitated in the colored hulless barley, but not in the white cultivar. With the exception of BQ_2, more flavonoids were up-regulated in the colored hulless barley compared to the white cultivar. These changes may be responsible for the observed variability in hulless 
barley color, including increased amounts of certain phenolic acids or flavonoids. The result was similar to the conclusion of a previous study in colored barley germplasm that found that seed coat color was related to the synthesis of anthocyanins (Kim et al. 2007; Shoeva et al. 2016), though we primarily detected flavonoids. In conclusion, colored hulless barley cultivars are rich in flavonoids and possess specific metabolite profiles that differ from white hulless barley, and these flavonoids also vary between the different colored cultivars. These findings improve our understanding of the metabolic mechanisms and functional health value associated with different seed coat colors in different hulless barley cultivars.

\section{Declarations}

This work was supported by funds from the National Key R \& D Program of China (2018YFD 1000705 /2018YFD1000700), the China Agriculture Research System (CARS-05) and the International Science and Technology Cooperation in Sichuan Province of China (Grant No. 2021YFH0113), and the Double Support Program for Discipline Construction of Sichuan Agricultural University in China.

\section{CONFLICT OF INTEREST STATEMENT}

The authors declare that they have no conflict of interest.

\section{References}

1. Abdel-Aal E, Choo TM, Dhillon S, Rabalski I (2012) Free and Bound Phenolic Acids and Total Phenolics in Black, Blue, and Yellow Barley and Their Contribution to Free Radical Scavenging Capacity. Cereal Chem 89(4):198-204

2. Abdel-Aal el-SM, Hucl P (2003) Composition and Stability of Anthocyanins in Blue-Grained Wheat. Food Chem, 51(8), 2174-2180

3. Abdel-Aal ESY, Rabalski JC, I (2006) Anthocyanin Composition in Black, Blue, Pink, Purple, and Red Cereal Grains. Food Chem 54:, 4696-4704

4. Badbro JS( (1993) Genetic control of flavonoid biosynthesis in barley. Hereditas 119(2):187-204

5. Bellido GG, \& B; T. (2009). Anthocyanin Composition and Oxygen Radical Scavenging Capacity (ORAC) of Milled and Pearled Purple, Black, and Common Barley. Agric Food Chem, 2009, 57(3): 1022-1028

6. Carlos G, Fraga BHC, Ronald J, Moore, Zink EM (2010) Signature-Discovery Approach for Sample Matching of a Nerve-Agent Precursor Using Liquid Chromatography-Mass Spectrometry, XCMS, and Chemometrics. Anal Chem 82(10):4165-4173

7. Chen W, Gong L, Guo Z, Wang W, Zhang H, Liu X,.. . Luo J (2013) A novel integrated method for largescale detection, identification, and quantification of widely targeted metabolites: application in the study of rice metabolomics. Mol Plant 6(6):1769-1780. doi:10.1093/mp/sst080

8. Dawson IK, Russell J, Powell W, Steffenson B, Thomas WTB, Waugh R (2015) Barley: a translational model for adaptation to climate change. New Phytol 206(3):913-931. doi:10.1111/nph.13266 
9. Dong X, Chen W, Wang W, Zhang H, Liu X, Luo J (2014) Comprehensive profiling and natural variation of flavonoids in rice. J Integr Plant Biol 56(9):876-886. doi:10.1111/jipb.12204

10. Fernie AR, Schauer N (2009) Metabolomics-assisted breeding: a viable option for crop improvement? Trends Genet 25(1):39-48. doi:10.1016/j.tig.2008.10.010

11. Ge X, Jing L, Zhao K, Su C, Zhang B, Zhang Q,.. . Li W (2021) The phenolic compounds profile, quantitative analysis and antioxidant activity of four naked barley grains with different color. Food Chem 335:127655. doi:10.1016/j.foodchem.2020.127655

12. Harlan HV (1914) Some distinctions in our cultivated barleys with reference to their use in plant breeding. U S Dept Agric Bul, 137

13. Idehen E, Tang Y, Sang S (2017) Bioactive phytochemicals in barley. J Food Drug Anal 25(1):148161. doi:10.1016/j.jfda.2016.08.002

14. Jung WY, Lau O, O’Keefe S-MC, Odell DP, Fader J, McGonigle G, B (2000) Identification and expression of isoflavone synthase, the key enzyme for biosynthesis of isoflavones in legumes. Nat. Biotechnol., 2000, 18, 208

15. Khakimov B, Bak S, Engelsen SB (2014) High-throughput cereal metabolomics: Current analytical technologies, challenges and perspectives. J Cereal Sci 59(3):393-418. doi:10.1016/j.jcs.2013.10.002

16. Khlestkina E (2013) The adaptive role of flavonoids: Emphasis on cereals. Cereal Research Communications 41(2):185-198. doi:10.1556/crc.2013.0004

17. Kim MJ, Hyun JN, Park KJA, Kim JC, M. Y., \& \& Kim JG et al (2007) Relationship between Phenolic Compounds, Anthocyanins Content and Antioxidant Activity in Colored Barley Germplasm. Agric Food Chem, 2007, 55: 4802-4809

18. León K, Mery D, Pedreschi F, León J (2006) Color measurement in L*a*b* units from RGB digital images. Food Res Int 39(10):1084-1091. doi:10.1016/j.foodres.2006.03.006

19. Lin S, Guo H, Gong JDB, Lu M, Lu M-Y, Wang L,... Wu D-T (2018) Phenolic profiles, $\beta$-glucan contents, and antioxidant capacities of colored Qingke (Tibetan hulless barley) cultivars. J Cereal Sci 81:6975. doi:10.1016/j.jcs.2018.04.001

20. Markou MT, Kassomenos $P$ (2010) Cluster analysis of five years of back trajectories arriving in Athens, Greece. Atmos Res 98(2-4):438-457. doi:10.1016/j.atmosres.2010.08.006

21. Ramakrishna R, Sarkar D, Schwarz P, Shetty K (2017) Phenolic linked anti-hyperglycemic bioactives of barley (Hordeum vulgare L.) cultivars as nutraceuticals targeting type 2 diabetes. Ind Crops Prod 107:509-517. doi:10.1016/j.indcrop.2017.03.033

22. Roljević Nikolić S, Kovačević D, Oljača D, Šeremešić A S (2020) Morphological and productive characteristics of hulless barley in organic farming. Ratar Povrt 57(1):27-34

23. Aastrup S, Outtrup H, Erdal K (1984) Location of the proanthocyanidins in the barley grain. Carlsberg Res Commun, 1984, 49(1):105-109 
24. Shen Y, Hu C, Zhang H, Jiang H (2018) Characteristics of three typical Chinese highland barley varieties: Phenolic compounds and antioxidant activities. J Food Biochem, 42(2). doi:10.1111/jfbc. 12488

25. Shoeva OY, Mock HP, Kukoeva TV, Borner A, Khlestkina EK (2016) Regulation of the Flavonoid Biosynthesis Pathway Genes in Purple and Black Grains of Hordeum vulgare. PLoS One 11(10):e0163782. doi:10.1371/journal.pone.0163782

26. Suriano S, lannucci A, Codianni P, Fares C, Menga V, Russo M,.. . Troccoli A (2020) Carotenoids and tocols content in genotypes of colored barley. J Cereal Sci, 96. doi:10.1016/j.jcs.2020.103110

27. Wang S, Tu H, Wan J, Chen W, Liu X, Luo J, Zhang H (2016) Spatio-temporal distribution and natural variation of metabolites in citrus fruits. Food Chem 199:8-17. doi:10.1016/j.foodchem.2015.11.113

28. Yan XY, Li JN, Fu FY, Jin MY, Chen L, Liu LZ (2009) Co-location of seed oil content, seed hull content and seed coat color QTL in three different environments in Brassica napus L. Euphytica 170(3):355364. doi:10.1007/s10681-009-0006-5

29. Yang XJ, Dang B, Fan MT (2018) Free and Bound Phenolic Compound Content and Antioxidant Activity of Different Cultivated Blue Highland Barley Varieties from the Qinghai-Tibet Plateau. Molecules, 23(4). doi:10.3390/molecules23040879

30. Yao X, Wu K, Yao Y, Bai Y, Ye J, Chi D (2018) Construction of a high-density genetic map: genotyping by sequencing (GBS) to map purple seed coat color (PSC) in hulless barley. Hereditas 155:37. doi:10.1186/s41065-018-0072-6

31. Zeng $X$, Long $H$, Wang Z, Zhao S, Tang Y, Huang Z,... Tashi N (2015) The draft genome of Tibetan hulless barley reveals adaptive patterns to the high stressful Tibetan Plateau. Proc Natl Acad Sci U S A 112(4):1095-1100. doi:10.1073/pnas.1423628112

32. Zeven AC (1991) Wheats with purple and blue grains: a review. Euphytica 56:43-258

33. Zhang G, Xue W, Dai J, Xu Q, Wang Y, Yuan H,.. . Nyima T (2019) Quantitative proteomics analysis reveals proteins and pathways associated with anthocyanin accumulation in barley. Food Chem 298:124973. doi:10.1016/j.foodchem.2019.124973

\section{Figures}



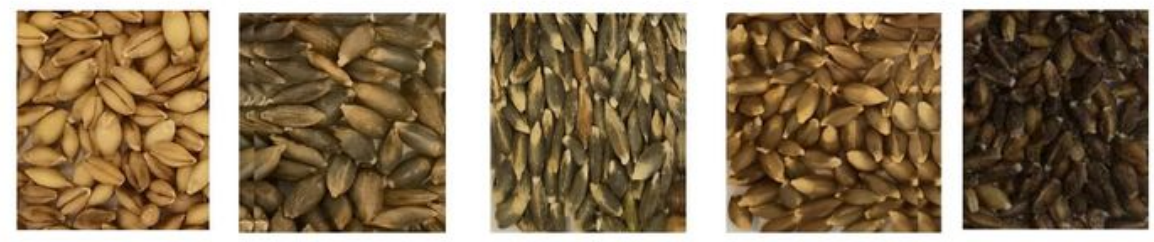

A

\section{Tree plot}

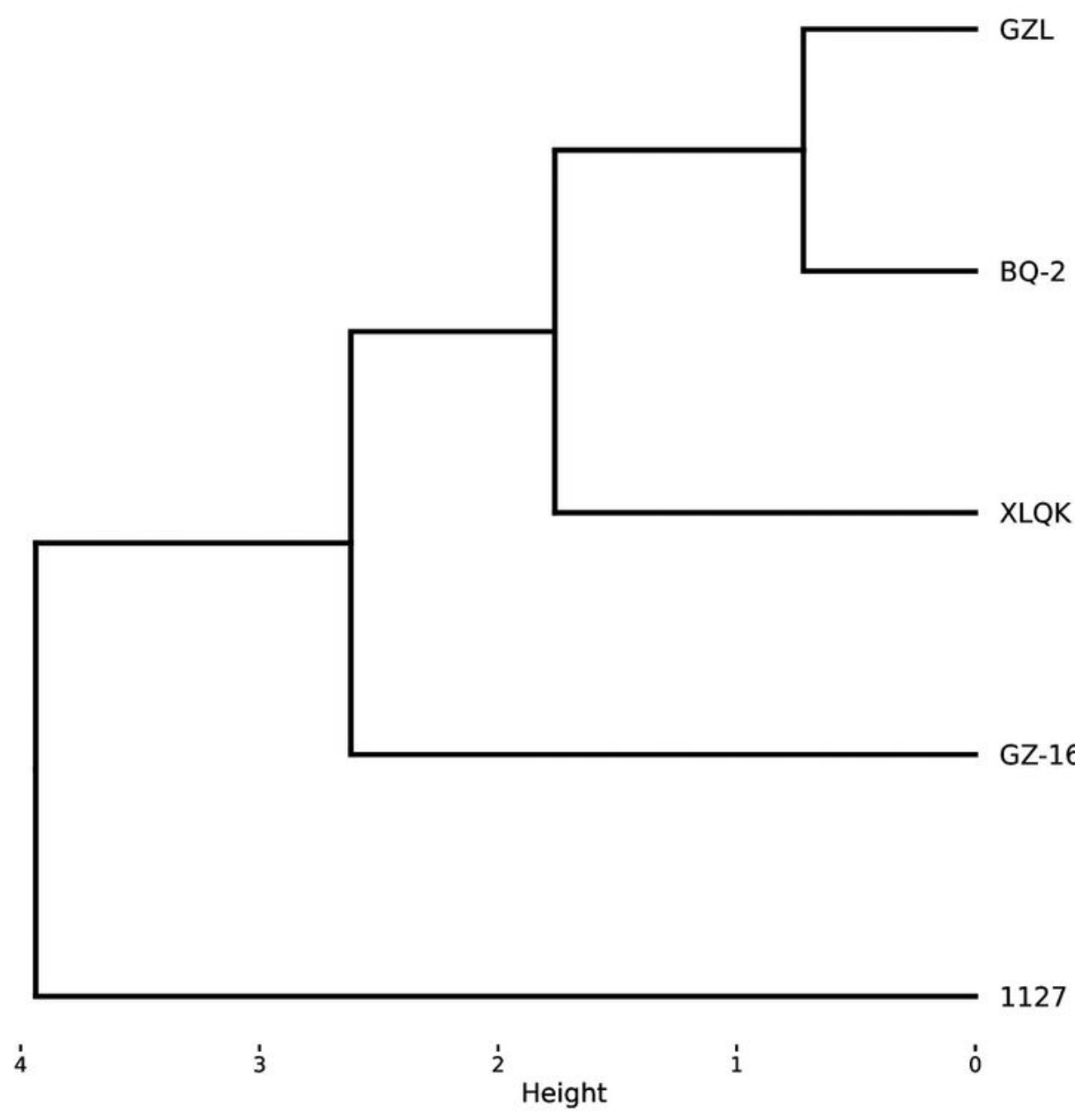

B

Figure 1

A Grain coat color of the five hulless barley varieties. B Clustering dendrogram of the color difference values of the hulless barley cultivars. 


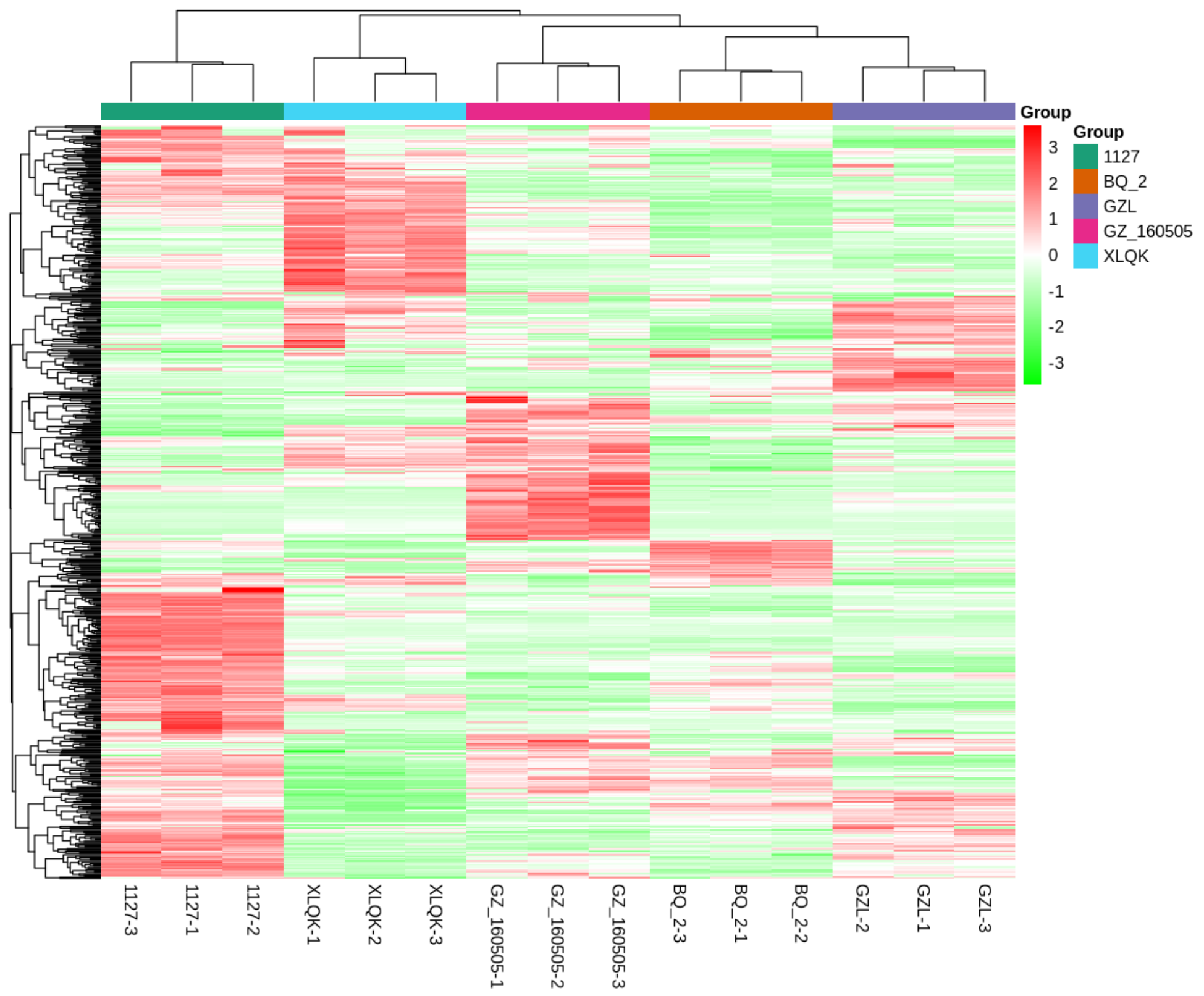

Figure 2

Metabolic profiles reflected using a clustering heatmap. The content of each metabolite was normalized to complete linkage hierarchical clustering. Green and red colors indicate low and high abundances, respectively. 
2D PCA Plot

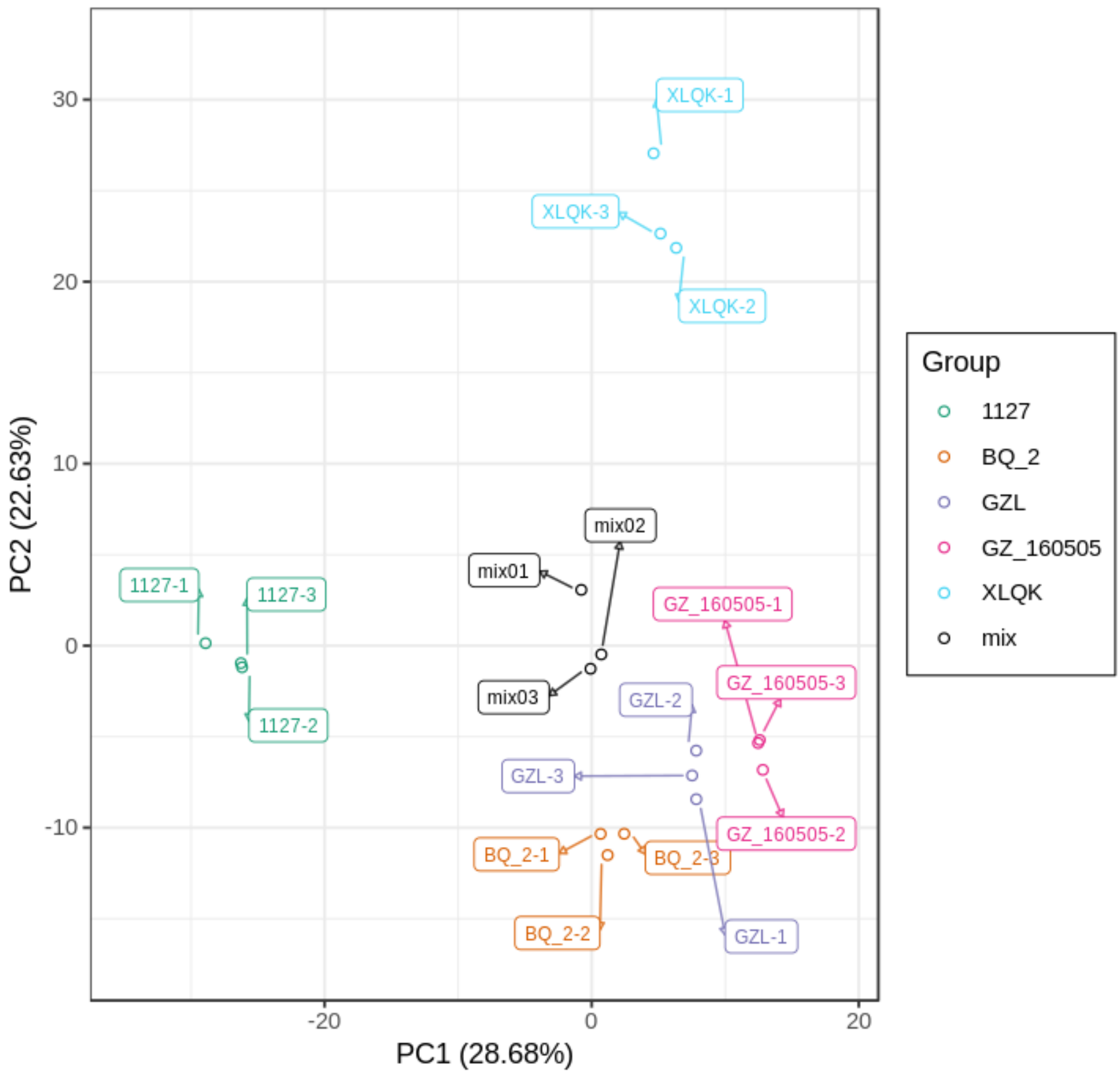

Figure 3

Principal component analysis (PCA) score plots of the five hulless barley cultivars. 


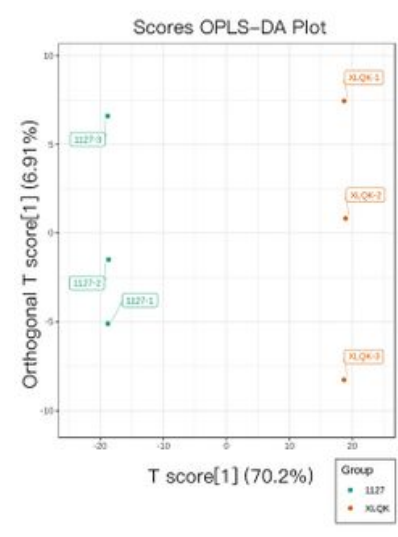

A

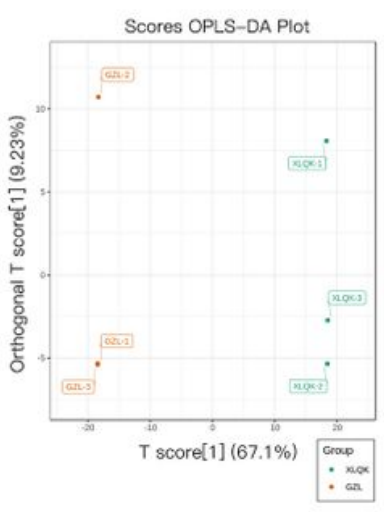

$\mathrm{F}$



B

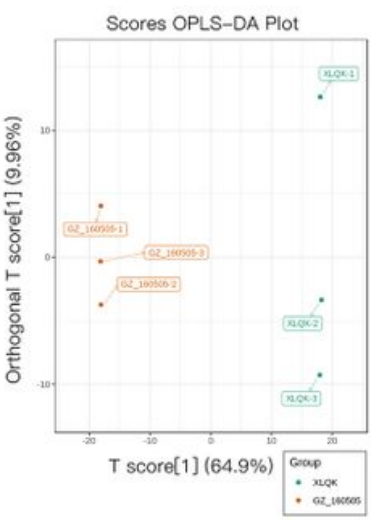

G

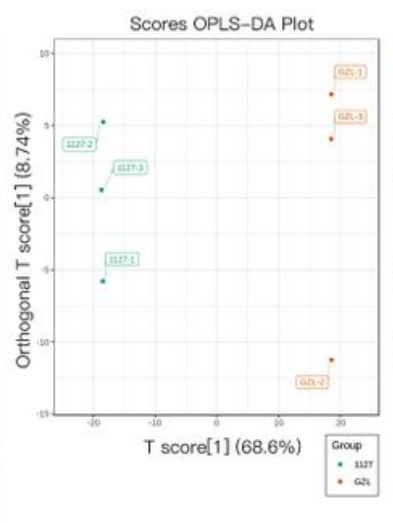

C



$\mathrm{H}$

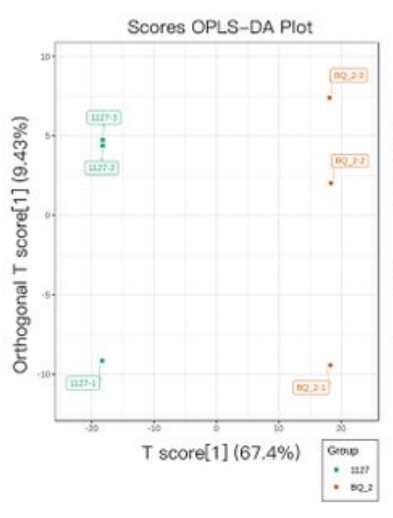

D
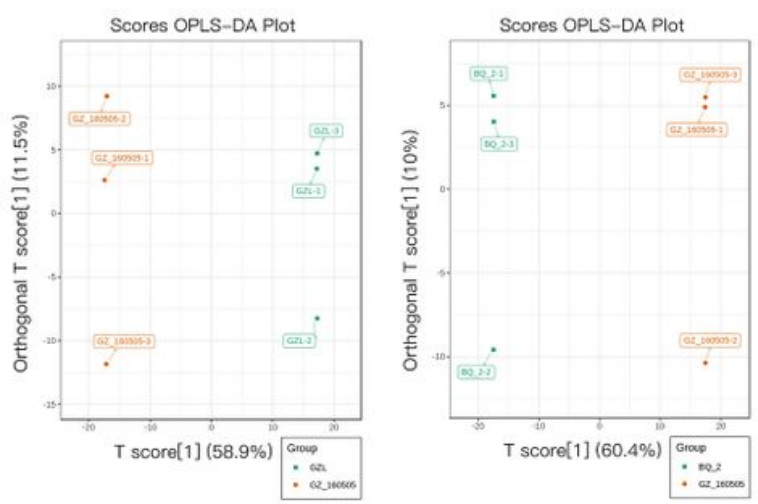

J

\section{Figure 4}

Differential metabolite analysis using orthogonal least squares-discriminant analysis (OPLS-DA). (A-J) OPLS-DA model plots for the comparison group of 1127 vs. XLQK, 1127 vs. GZ_160505, 1127 vs. GZL, 1127 vs. BQ_2, XLQK vs. BQ_2, XLQK vs. GZL, XLQK vs. GZ_160505, GZL vs. BQ_2, GZL vs. GL_160505, and BQ_2 vs. GL_160505. 


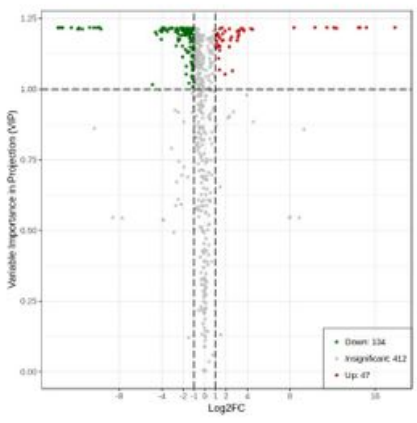

A. 1127_vs_BQ_2

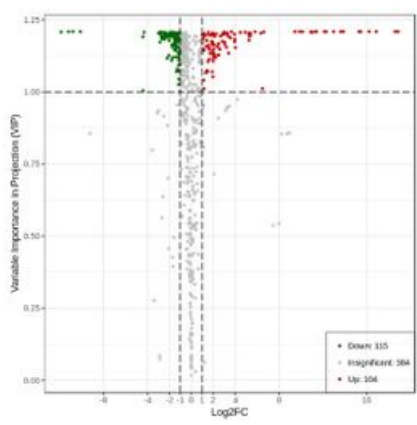

B. 1127_vs_GZ_160505

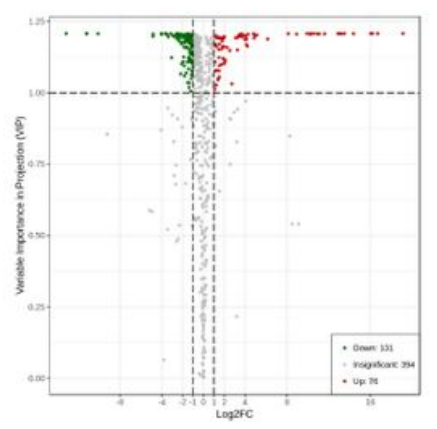

C. 1127 vs_GZL

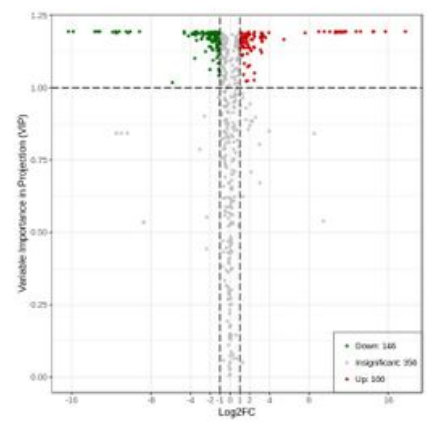

D. 1127_vs_XLQK

venn


$\mathrm{F}$

$\mathrm{E}$

Figure 5

Differential metabolite analysis of colored hulless barley compared with white hulless barley. A-D: The volcano plot shows the differential metabolite abundance levels between BQ_2, GZ_160505, GZL, and XLQK compared with 1127. Green dots represent down-regulated differentially abundant metabolites; red dots represent up-regulated differentially abundant metabolites; and black dots represent metabolites detected but that are not significantly different. E: Venn diagram showing the overlapping and unique differential metabolites among the comparison groups; F: Venn diagram showing the overlapping and unique metabolites among the comparison groups. 


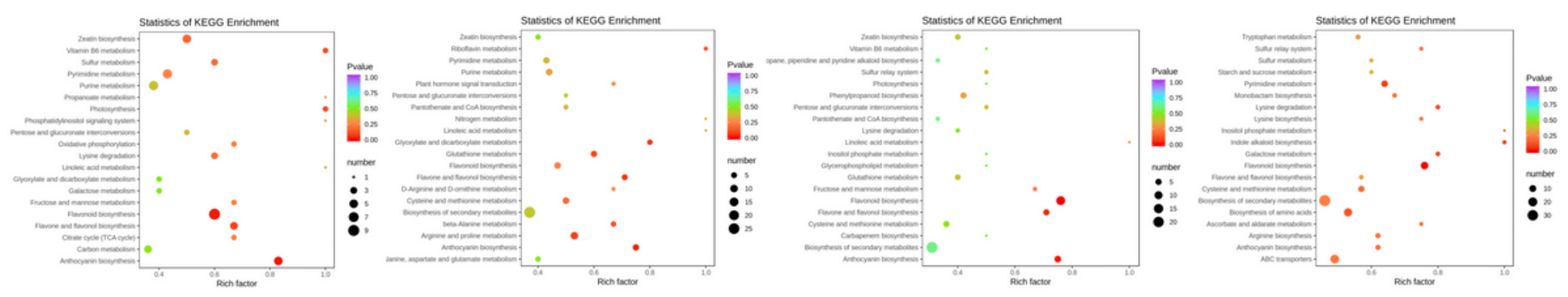

\section{Figure 6}

Enrichment map of the differential metabolites to distinct KEGG pathways. Differential metabolites in BQ_2 (A), GZL (B), GZ_160505 (C), and XLQK (D) compared with those in 1127 were mapped to distinct metabolic pathways. 